\title{
DTI Volume Rendering Techniques for Visualising the Brain Anatomy
}

\author{
Burkhard Wünsche, Jarno van der Linden, Nathan Holmberg \\ Department of Computer Science, University of Auckland, Private Bag 92019, Auckland, New \\ Zealand
}

\begin{abstract}
Over the past few years Diffusion Tensor Imaging (DTI) has become an increasingly popular method for imaging the brain anatomy and diagnosing a variety of neurodegenerative diseases. Unfortunately the size and multi-dimensional nature of diffusion tensor data sets makes it difficult to understand them. We use illuminated streamlines to compute high quality dense 3D visualisations of the 3D nerve fibre structure. Nerve fibres are extracted using a numerical integration technique and a fuzzy classifier which represents the probability that a sample point represents grey matter, white matter or Cerebral Spinal Fluid (CSF). We present two novel methods which improve the perception of the 3D arrangements of fibre tracts. The first method is a hardware accelerated algorithm which represents fibres as semi-transparent tubes with emphasised silhouettes. Because of the semi-transparent nature of the tubes inside structures are revealed. The enhancement of tube silhouettes improves the identification of individual fibre tracts and their 3D arrangement. The second method uses direct volume rendering and multiple colour and transparency look-up tables to represent the directional information of the nerve fibre structure and other tissue types simultaneously. The method can be used to represent finer details depending on the resolution of the noise texture employed. Depending on the choice of the opacity transfer functions fibre tracts can be represented semi-transparent or nearly opaque.
\end{abstract}

Key words: Biomedical Visualisation, Tensor Field Visualisation, Diffusion Tensor Imaging, Brain Anatomy, Nerve Fibre Tracking, Line Integral Convolution, Direct Volume Rendering

Email addresses: burkhard@cs.auckland.ac.nz (Burkhard Wünsche), jarno@tufflittleunit.com (Jarno van der Linden), n.holmberg@gmail.com (Nathan Holmberg).

URLs: http://www.cs.auckland.ac.nz/ burkhard (Burkhard Wünsche), http://www.cs.auckland.ac.nz/ ${ }^{\sim}$ jvan006 (Jarno van der Linden). 


\section{Introduction}

Diffusion tensor imaging (DTI), also known as diffusion-weighted MRI imaging (DW$\mathrm{MRI}$ ), is used to measure the intrinsic properties of water diffusion in the brain by an orientation invariant quantity, the diffusion tensor $\mathbf{D}[1,2]$. In the brain DTI can be used to differentiate three types of structures. Fluid filled compartments are characterised by a very high isotropic diffusion, i.e., the diffusion is similar in all directions and the mean diffusivity is high. In contrast nerve fibres restrict the diffusion to one direction only due to the presence of cell membranes and myelin sheaths surrounding the axons. Fibre tracts, consisting of millions of parallel nerve fibres, are therefore identified as areas of a high anisotropic diffusion. The orientation of such fibre tracts is determined from the principal directions of the diffusion tensor. Finally gray matter is characterised by a low and nearly isotropic diffusion since the water diffusion is restricted in all directions due to cell membranes of intermingled cell bodies and their surrounding neuralgia. Consequently DTI can be used to gain in vivo information about the anatomy, micro-structure and physiology of the brain [3].

Three dimensional diffusion tensor data sets are usually visualised by reconstructing nerve fibre tracts and representing them with streamlines or streamtubes. Kindlmann et al. [4] visualise the $3 \mathrm{D}$ geometry of the diffusion tensor field using a direct volume rendering technique with the colour, lighting and opacity assignment governed by the underlying tensor field. Fibre tracts show up as regions of slowly varying saturated colour, however, the 3D fibre tract geometry is difficult to perceive. Recently a new method has been presented which represents fibre tracts as thin threads with a surrounding halo [5]. Direct volume rendering the resulting data sets gives excellent results.

\section{Methods}

Streamlines are created by integrating along the maximum diffusion direction using a $4^{\text {th }}$ order Runge-Kutta method. The seed points from which streamlines grow are defined by computing the probability that a sample point is within a nerve fibre tract. This probability determines the number of potential seed points generated in the vicinity of a sample point. Using this method we create around 9500 start points from 1 million voxels. The probability function used in this method is the weighted product of two separate probability functions for the anisotropy and the mean diffusivity of the tensor field, which are based on knowledge about the water diffusion in different types of brain tissue as explained in section 1 (see figure 1). Since diffusion properties typical for nerve fibre tracts can also be registered due to noise or eddy currents in fluid filled compartments 
we test at each step whether the probability of being within a nerve fibre tract exceeds $60 \%$. In addition the final fibre tract must exceed a minimum length $(8.2 \mathrm{~mm})$ and its maximum allowed curvature is controlled.
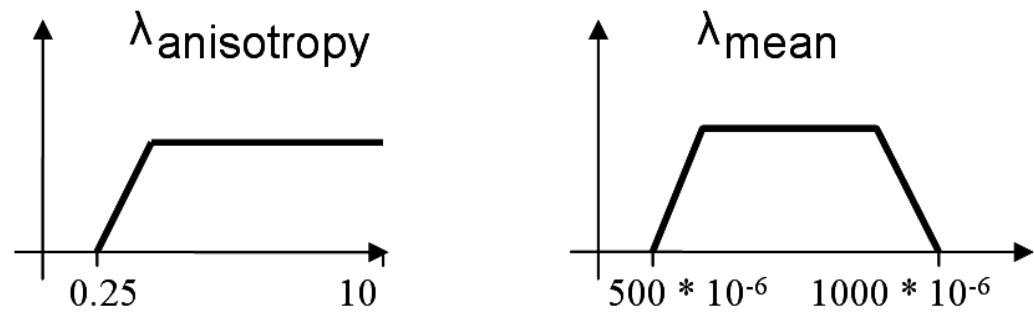

Fig. 1. The probability function for detecting nerve fibre tracts is the product of probability functions with respect to the diffusion anisotropy (left) and the mean diffusivity (right).

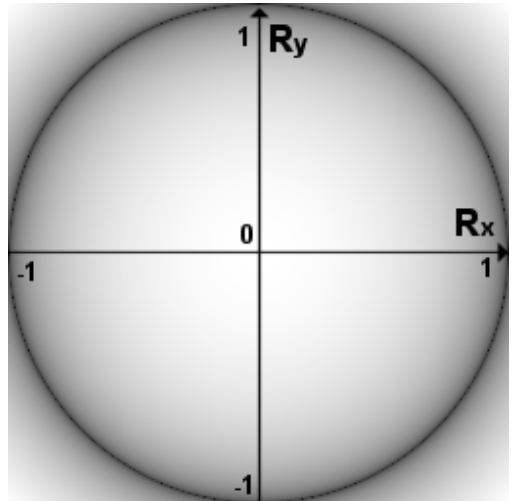

Fig. 2. A texture used with reflection mapping for edge enhanced transparency.

Representing fibre tracts by simple streamlines results in images where the exact 3D geometry is difficult to understand since depth cues due to shading and overlay are missing. Streamlines can be illuminated using a Phong illumination model and replacing the missing surface normals with planes of possible normals which are orthogonal to the streamline. The technique can be implemented efficiently in hardware by precomputing a texture which encodes the illumination obtained for different view points, light directions and tangents of the streamline [6]. An alternative way to illuminate streamlines is to create streamtubes by fitting a thin Gouraud shaded cylinder around them.

Using streamtubes can result in visual cluttering when the underlying streamlines are densely packed together. The resulting images also often suffer from aliasing effects which makes the interpretation of the 3D geometry difficult. These problems can be reduced by decreasing the density of streamtubes or removing unwanted streamtubes. However, this can result in the loss of important information and often requires user interaction in order to obtain satisfactory results. Making the streamtubes instead semi-transparent allows inner structure to be revealed without requiring user interaction and changes to the streamtubes.

Polygon renderers such as OpenGL implement transparency by drawing polygons using a partial alpha value, letting some of the background colour blend with the polygon colour. The result lacks real-world effects such as attenuation and reflection at small incidence angles. Transparency can also reduce the impact of shading. To improve the perception of transparent streamtubes we enhance the silhouette edges by varying the amount of 
transparency as a function of incidence angle. This approximates the apparent change in transparency that happens towards the silhouette edges of real objects such as glasses. The incidence angle is given by the angle between the normal at a point on the streamtube, and the ray from that point to the eye. We accelerate shading in hardware by precomputing a texture which encodes the transparencies as functions of the incidence angle. We use the GL_REFLECTION_MAP texture coordinate generation method which computes texture coordinates using the $x$ and $y$ components of the reflection vector $\mathbf{R}$ in eye coordinates. These range from 0 for face-on points, to \pm 1 for points with a normal near $45^{\circ}$, and back to 0 for points on the silhouette. We use this to our advantage, since this causes an increase in transparency for points near the silhouette edges which softens the appearance of the edges and reduces aliasing effects. Converting the reflection vector coordinate range $[-1 . .1]$ to the texture coordinate range $[0 . .1]$ is done through a simple scale and translate in the texture matrix. An example of the texture used is shown in figure 2. The shape of the gradient can be adjusted to, for example, make silhouette edge stronger, or make the streamtubes appear more opaque.

We direct volume render nerve fibre tracts by using an algorithm similar to Seed LIC [7] whereby the seed points used are identical to those in the streamline visualisation discussed above. The seed points are distributed based on the probability that a point is within the region of interest (ROI). This means that the overhead associated with using Fast LIC and ensuring a certain number of hits everywhere (even outside the ROI) is avoided ensuring the generation time is measured in seconds rather than hours. A further advantage of this method is that the generation of streamlines performed for previous visualisations can be reused ensuring that the nerve fibre structures are tracked appropriately with the necessary physiological constraints being enforced. The LIC texture generation is similar to that for the Seed LIC algorithm. Further improvements were obtained by using two data sets to define the transfer functions. One data set contains the Line Integral Convolution (LIC) texture which encodes the direction information of nerve fibre structures. The second data set contains information about the expected probability that a particular voxel lies within the nerve fibre structure which is estimated by the number of times the associated cell has been hit by field lines during the generation of the (LIC) texture.

\section{Results}

The top row of figure 3 compares various streamline based visualisation methods. Simple non-illuminated streamlines work well for individual fibre tracts and large scale views but perform poorly when examing complex fibre tract arrangements closely. Using streamtubes improves the 3D perception but leads to visual cluttering because of their larger spatial extend. Furthermore valuable depth cues due to overlay are lost and the tubes 
are often misinterpreted by laymen who perceive them as the actual representation of nerves. In contrast illuminated streamlines, which according to our knowledge have not been previously used for nerve fibre tract visualisation, can be used to represent finer 3D details using an illumination model which is popular for hair rendering.

Our first new method represents nerve fibres using tube-like structures, but because of the semi-transparent nature of the tubes inside structures are now revealed. Emphasising the silhouettes improves the 3D perception and accentuates nerve fibre tracts in the front as illustrated in the two images in the bottom left of figure 3. Our second new method can be used to represent finer details depending on the resolution of the noise texture employed. By varying the opacity transfer functions fibre tracts can be represented semitransparent or nearly opaque. Interactively varying the opacity improves the $3 \mathrm{D}$ perception even further. A disadvantage of this method is that for high transparency values the 3D arrangement of fibre tracts is not as clear as for the previously described techniques.
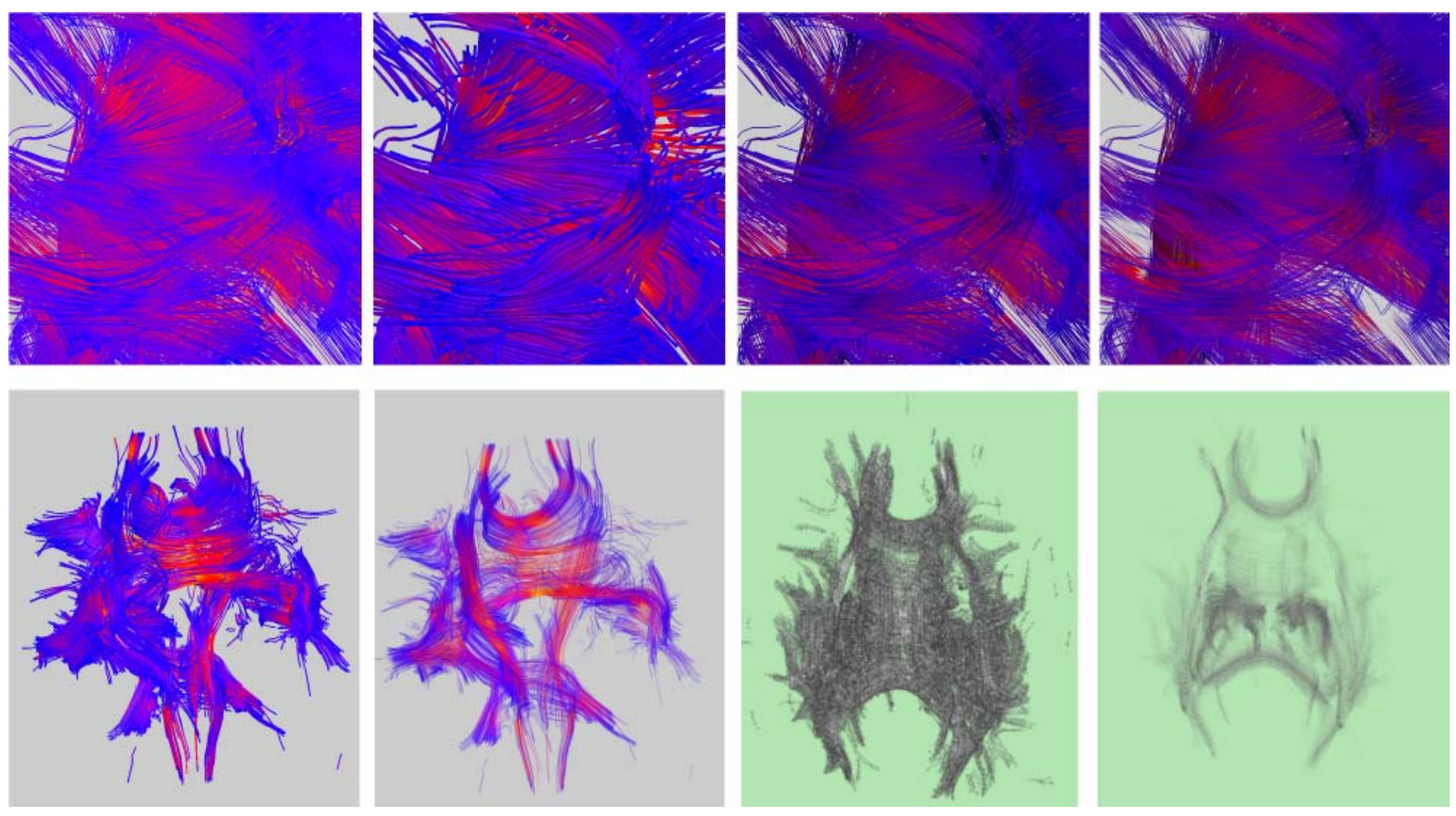

Fig. 3. Top row (from left to right): a part of the internal capsule visualised using streamlines, streamtubes, illuminated streamlines without and with transparencies. Bottom left: The nerve fibre structure in the brain visualised normal streamtubes and edge enhanced transparent streamtubes. Bottom right: Direct volume rendering of an LIC texture using a standard opacity transfer function (left) and using our fuzzy classifier (right). 


\section{Conclusion}

Visualising the diffusion tensor field in the brain gives an in vivo view of anatomical structures which was previously unobtainable. Nerve fibres are extracted using a numerical integration technique and a fuzzy classifier which represents the probability that a sample point represents grey matter, white matter or Cerebral Spinal Fluid (CSF). Using illuminated streamlines makes it possible to obtain high quality dense 3D visualisations of the 3D nerve fibre structure. We introduced a novel hardware accelerated algorithm which represents fibre tracts as semi-transparent tubes with emphasised silhouettes which improves the identification of individual tubes and their 3D arrangement. A second method uses a direct volume rendering technique which represents fibre tracts by a line integral convolution texture and uses an opacity transfer functions based on a fuzzy tissue classier which makes it possible to represent fibre tracts from semi-transparent to nearly opaque.

\section{References}

[1] P. J. Basser, J. Mattiello, D. L. Bihan, MR diffusion tensor spectroscopy and imaging, Biophysical Journal 66 (1994) $259-267$.

[2] P. J. Basser, C. Pierpaoli, Microstructural and physiological features of tissues elucidated by quantitative-diffusion-tensor MRI, Journal of Magnetic Resonance Series B 111 (3) (1996) $209-219$.

[3] B. C. Wünsche, R. Lobb, The 3D visualization of brain anatomy from diffusion-weighted magnetic resonance imaging, in: Proceedings of GRAPHITE 2004, ANZGRAPH and SEAGRAPH, 2004, pp. $74-83$.

[4] G. Kindlmann, D. Weinstein, D. Hart, Strategies for direct volume rendering of diffusion tensor fields, IEEE Transactions on Visualization and Computer Graphics 6 (2) (2000) 124 -138 .

[5] A. Wenger, D. F. Keefe, S. Zhang, D. H. Laidlaw, Interative volume rendering of thin thread structures within multivalued scientific data sets, IEEE Transactions on Visualization and Computer Graphics 10 (6) (2004) $664-672$.

[6] D. Stalling, M. Zöckler, H.-C. Hege, Fast display of illuminated field lines, IEEE Transactions on Visualization and Computer Graphics 3 (2) (1997) 118 - 128.

[7] A. Helgeland, O. Andreassen, Visualization of vector fields using seed LIC and volume rendering, IEEE Transactions on Visualization and Computer Graphics 10 (6) (2004) 673 -682 . 